\title{
Tumor cells may circulate in medullary thyroid cancer patients independently of serum calcitonin
}

\section{Dear Editor,}

Early detection of tumor relapse is a major issue in patients with medullary thyroid carcinoma. Calcitonin has been reported as a sensitive and accurate marker for recurrence of medullary thyroid carcinoma after thyroidectomy. Recent evidence nevertheless reveals pitfalls in calcitonin immunoassays due to the presence of heterophilic antibodies or macroaggregates (i.e. falsely increased values or macrocalcitonin) (Alves et al. 2016). Calcitonin can also remain undetectable despite metastasis of rare tumor cells in thyroidectomized patients. In this context, we designed a sensitive and specific technique to identify calcitoninpositive circulating tumor cells (CTC) in medullary thyroid carcinoma. We demonstrate that calcitonin-positive CTCs are present in the peripheral blood of medullary carcinoma patients following complete thyroidectomy. Unexpectedly, the presence of CTCs could be identified up to 12 years after surgery even in the absence of high levels of serum calcitonin.

Incidence of thyroid cancer significantly increased in the past several decades (Kuo et al. 2016). Tumors most frequently arise from follicular epithelial cells to generate papillary, follicular or anaplastic thyroid cancers. Medullary thyroid carcinoma (MTC) initiates in neuroendocrine $\mathrm{C}$ cells accounting for approximately 4-5\% of all thyroid cancers (Xing 2013). Since MTCs are unresponsive to radioiodine therapy, surgical resection of the tumor is the first-line treatment. With 10-year survival rates of about $75 \%$, thyroidectomy is generally the most adequate therapeutic option. The main issue in MTC management is prediction of tumor relapse. The serum concentration of calcitonin is a specific and cost-effective biomarker that adequately predicts tumor relapse or metastasis. Indeed, the doubling time of serum calcitonin is a reliable prognostic marker used in routine management of MTC (Ito et al. 2016). Recent evidence has nevertheless challenged calcitonin-based immunoassays due to the presence of heterophilic antibodies in patient serum or cross-reactivity with procalcitonin and related peptides (Alves et al. 2016). These issues should be solved to improve post-operative prognosis. When calcitonin levels exceed $500 \mathrm{pg} / \mathrm{mL}$, distant metastases are almost always identified by radiographic imaging. In contrast, when serum calcitonin is low, the risk of persistent or recurrent residual disease is low. A challenge in decisionmaking occurs in presence of intermediate calcitonin levels $(150 \mathrm{pg} / \mathrm{mL})$ or when concentrations are persistently increased but unchanged over time. In these conditions, local or distant metastases are difficult to detect by radiographic imaging and require additional and often expensive tests during follow-up. In this context, we investigated another approach based on the identification of thyroid-specific CTCs. CTCs are cells that detach from the primary or metastatic tumor and intravasate into the blood stream (Yu et al. 2011). Since CTCs travel in the blood to develop a secondary tumor at a distant site, their frequency can be used as a prognostic marker for relapse. Currently, main issues in CTC enumeration pertain to their extreme scarcity compared to blood cells and to phenotypic changes (epithelial-to-mesenchymal transition (EMT)). A series of tests have been developed based on cell size fractionation, microfluidics or antigen capture. In particular, the CellSearch (Veridex) is a FDA-validated system that enumerates CTCs upon capture of EpCAM-positive epithelial cells in whole blood. If exceeding a defined threshold, CTC levels have been demonstrated to worsen prognosis of the situation (Yu et al. 2011). The principle of the technique is based on automated sorting of CTCs with an epithelial marker (EpCAM) and subsequent detection of CD45-negative, cytokeratin-positive nucleated cells. To identify CTCs 
in thyroid cancer, a series of 15 patients was analyzed by CellSearch (study \#2014/93 approved by the Ethics Committee of the CHU University Hospital, Liège, Belgium). In the majority of cases $(60 \%, 9$ out of 15$)$, no CTC was identified (Fig. 1A, B and Supplementary Table 1, see section on supplementary data given at the end of this article). Four subjects out of 15 (27\%) had 1 CTC in $7.5 \mathrm{~mL}$ of blood. The two remaining patients (2 out of 15) with medullary and papillary thyroid cancers displaying lung and lymph node metastases had 2 and 7 CTCs, respectively. As controls, CTCs were enumerated in cancers associated with high (small-cell lung cancer (SCLC)) and low (non-small-cell lung cancer (NSCLC)) cell counts. In accordance with previous studies (Krebs et al. 2011, Hou et al. 2012), CTCs were detected in all SCLC cases $(n=6)$ and in most NSCLC ( $n=41$ out of 67$)$ cases (Fig. 1B and Supplementary Table 1). These data thus indicate that EpCAM-positive CTCs are undetectable in most thyroid cancer patients validating and extending previous observations (Yu et al. 2016). Therefore, we set up another technique based on cell filtration (ScreenCell device) and calcitonin expression to identify CTCs in MTC. The reactivity of a FDA-approved antibody used in routine diagnosis (SP17) was first evaluated on three cell lines pertaining to the major thyroid cancer subtypes: TT (medullary), TPC-1 (papillary) and C643 (anaplastic). Immunofluorescence analysis showed that only TT cells expressed calcitonin, as expected (Fig. 1C and Supplementary Fig. 1). Immunohistochemistry of a thyroid biopsy further indicated that parafollicular $\mathrm{C}$ cells specifically stained positive for calcitonin (Supplementary Fig. 2). These experiments thus validate the specificity of the anti-calcitonin SP17 antibody.

Using the ScreenCell device, CTCs were isolated from peripheral blood of a patient with MTC and stained with SP17. Figure 1D illustrates a typical fluorescence scan of CTCs expressing calcitonin in the cytoplasm and containing a DAPI-stained nucleus (blue arrows). A major criterion to distinguish CTCs from leucocytes is their larger diameter (at least three-fold) that does not allow filter pore bypass. Although the filter pores yielded some background autofluorescence, the specificity of labeling was demonstrated by the presence of another cell lacking calcitonin expression (see * on Fig. 1D) and by spike-in experiments (Supplementary Fig. 3). In contrast, no calcitonin-positive CTCs could be detected in peripheral blood from four healthy volunteers (Supplementary Fig. 4).

Using these optimized experimental conditions, the presence of calcitonin-positive CTCs was investigated in a series of subjects with MTC. We selected nine patients at different TNM stages having undergone complete thyroidectomy and, except for \#9, lacking any evidence of other cancer (Table 1 for clinical and pathological characteristics). The calcitonin concentrations in the serum were above the basal level of the assay (i.e. $10 \mathrm{pg} / \mathrm{mL}$ ) and ranged from 48 to $10,600 \mathrm{pg} / \mathrm{mL}$, further validating the diagnosis. Calcitonin-positive CTCs were identified in all patients (from 1 to 7), except one (\#1). Interestingly, the CTC counts did not correlate with TNM classification as illustrated for example by patient \#1 at pT3N1bM1 lacking any detectable calcitonin-positive circulating cell while 5 CTCs were scored in subject \#7 at pT1N1bMx. Finally, the serum calcitonin concentrations did not correlate with CTC counts $\left(R^{2}=0.11272\right.$, Fig. 1E). In particular, the calcitonin levels were low in patients \#5, $\# 7$ and \#8 (70, 48 and $82 \mathrm{pg} / \mathrm{mL}$ ) while CTC counts were high ( $n=4,5$ and 7$)$, respectively.

We have thus set up a technique that combines the ScreenCell device and calcitonin labeling that very specifically identifies CTCs in MTC patients even after thyroidectomy. Compared to the FDA-cleared CellSearch, our experimental protocol has a series of advantages. First, the CellSearch enumerates CTCs by cell sorting using an epithelial marker (EpCAM) that is frequently lost due to EMT. Our data indicate that this approach is indeed inadequate for thyroid carcinoma due to lack of sensitivity. This conclusion is in agreement with a recent report showing that, even at metastatic stages, only 7 out of 18 MTC have more than 2 CTCs (Yu et al. 2016). In contrast, our method is able to identify calcitonin-positive CTCs in thyroidectomized patients considered to be in persistent disease. Secondly, calcitonin labeling specifically identifies CTCs derived from MTC excluding any other neoplasm such as breast cancer (patient \#9 in this study). Third, gentle cell filtration by ScreenCell is also compatible with microemboli that cannot be scored by CellSearch. Fourth, the protocol does not require any expensive equipment in addition to filtration columns.

The ScreenCell device and calcitonin labeling approach also has some disadvantages. Although visual inspection very specifically reveals fluorescent cells expressing calcitonin, validation of CTC phenotype requires the intervention of a trained pathologist. The technique also requires further automation to be compatible with routine diagnosis. Another limitation of the technique is that blood samples should be analyzed fresh or at least within a maximum of $92 \mathrm{~h}$. Despite these drawbacks, the ScreenCell device and calcitonin labeling methodology 
A

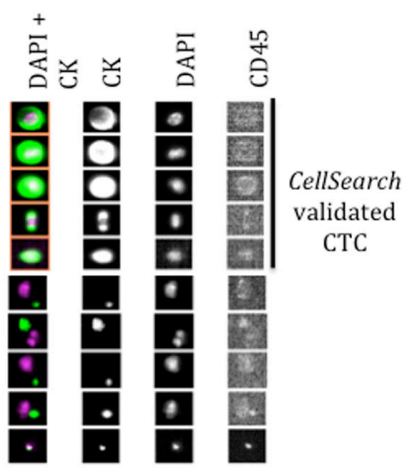

B

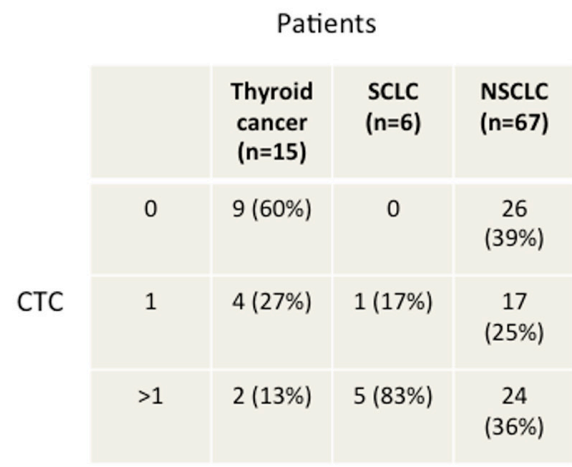

C

D

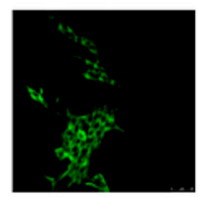

Calcitonin

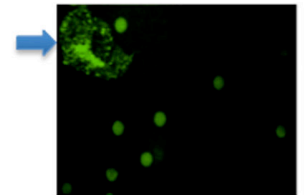

Calcitonin

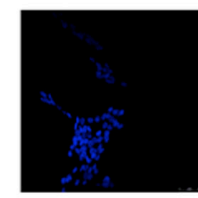

DAPI

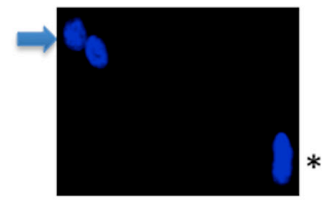

DAPI

E

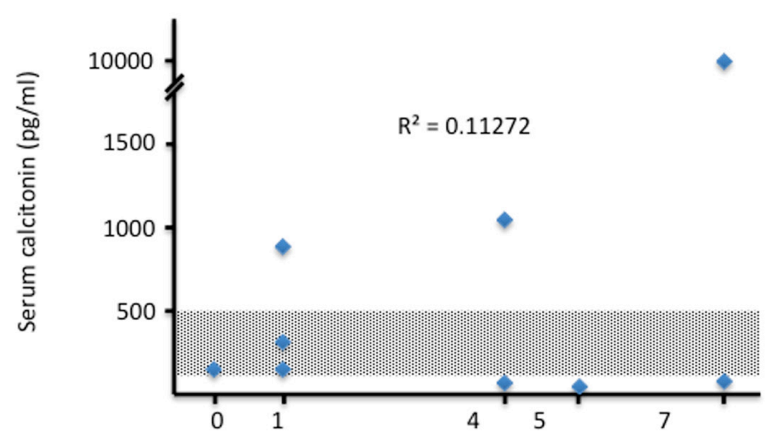

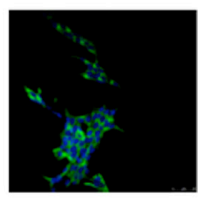

Overlay

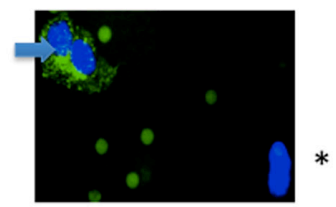

Overlay
Medullary cell line

(TT)

\section{Figure 1}

(A) Representative results of CTCs identified using the CellSearch system (Veridex). CTCs were isolated with an EpCAM antibody and labeled for cytokeratin (CK), nuclei (DAPI) and leukocyte common antigen (CD45) as described in the Supplementary data. (B) Summary of CTC counts in thyroid cancer, small-cell lung cancer (SCLC) and non-small-cell lung cancer (NSCLC). The numbers and percentages of patients without CTC (0), with one or with more than one are indicated. (C) The TT cell line derived from medullary thyroid carcinoma was labeled with SP17 anti-calcitonin antibody and visualized by fluorescence microscopy. (D) CTCs were isolated by size of epithelial tumor cells (ScreenCell device) from peripheral blood of a patient with medullary thyroid cancer. Cells were labeled and visualized as described in panel B. Arrows indicate calcitonin-positive CTCs (green) and their nuclei (DAPI in blue). (E) The number of calcitoninpositive CTCs was plotted against the concentration of serum calcitonin (pg/mL). $R^{2}$ is the correlation coefficient. can, as described, readily be set up in standard conditions of routine diagnosis.

Although the series of samples are limited, this report highlighted an unexpected and interesting observation: relatively high CTC counts can be detected despite low calcitonin levels in the serum of three patients (\#5, \#7 and \#8). Since CTC numbers correlate with the probability of tumor relapse, there is an intrinsic risk that prognosis of these subjects will worsen in the near future. Careful follow-up of these patients and further validation in larger cohorts will confirm this observation. This is particularly important because follow-up periods in our series are longer in the group of low CTC count (especially in cases 1, 2 and 4) compared to the group of high CTC number of CTC count (cases 5-9). Therefore, overall survival or diseasefree survival may show poorer in high CTC count group than in low CTC count group. Nevertheless, we believe that the presence of calcitonin-positive CTCs in the absence of significant levels of serum calcitonin should be considered to initiate additional tests during follow-up. There is indeed growing evidence that the presence of CTC correlates with poorer prognosis (Yu et al. 2016). Further follow-up experiments are required to assess whether prognosis depends on calcitonin-positive CTC counts. Since neither radiotherapy nor chemotherapy has demonstrated durable objective responses in patients with advanced MTC, early identification of tumor relapse is predicted to increase overall survival. 


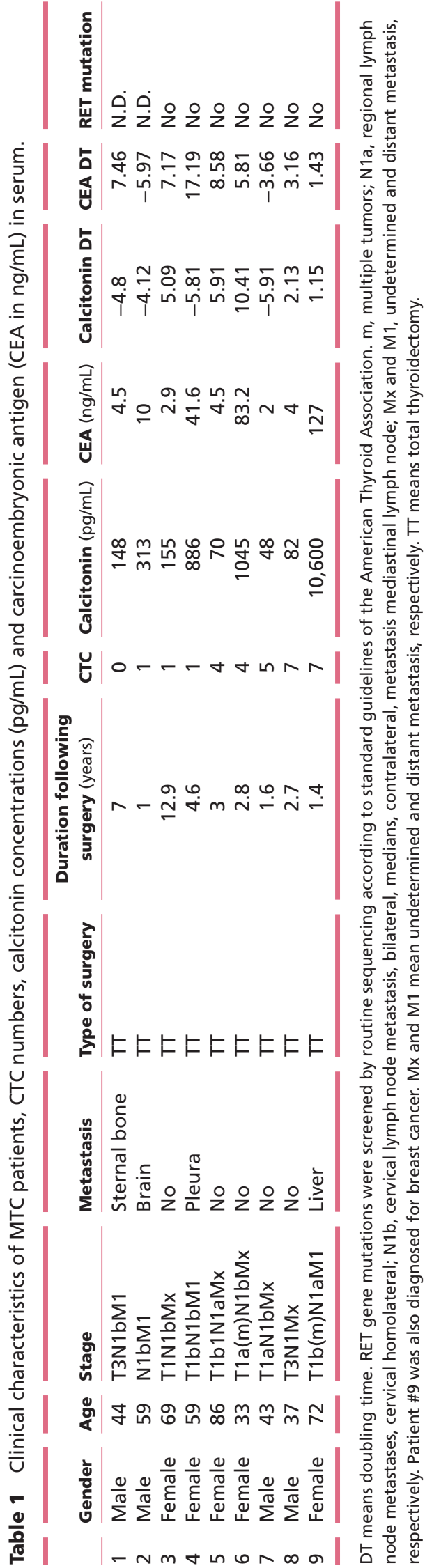

http://erc.endocrinology-journals.org https://doi.org/10.1530/ERC-18-0180
(C) 2018 Society for Endocrinology Published by Bioscientifica Ltd. Printed in Great Britain
Sathya Neelature Sriramareddy ${ }^{1,2}$

Etienne Hamoir ${ }^{3}$

Marcela Chavez ${ }^{4}$

Renaud Louis ${ }^{5}$

Albert Beckers 3

Luc Willems ${ }^{1,2}$

${ }^{1}$ Molecular and Cellular Epigenetics (GIGA),

University of Liege, Liège, Belgium

${ }^{2}$ Molecular Biology (TERRA), University of Liege, Gembloux, Belgium

${ }^{3}$ Endocrinology, University Hospital, Liège, Belgium

${ }^{4}$ Clinical Hematology, University Hospital,

Liège, Belgium

${ }^{5}$ Pneumology, University Hospital, Liège, Belgium

(Correspondence should be addressed to L Willems:

luc.willems@uliege.be)

Supplementary data

This is linked to the online version of the paper at https://doi.org/10.1530/ ERC-18-0180.

Declaration of interest

The authors declare that there is no conflict of interest that could be perceived as prejudicing the impartiality of this article.

\section{Funding}

This work received financial support from the 'Fonds National de la Recherche Scientifique' (FNRS), the Télévie, the Interuniversity Attraction Poles (IAP) Program 'Virus-host interplay at the early phases of infection' BELVIR initiated by the Belgian Science Policy Office, the Belgian Foundation against Cancer (FBC), the 'Centre anticancéreux près ULg' (CAC) and the 'Fonds Léon Fredericq' (FLF), the 'AgriculturelsLife' project of Gembloux Agrobiotech (GXABT), the 'ULg Fonds Spéciaux pour la Recherche' and the 'Plan Cancer' of the 'Service Public Fédéral'. S N S obtained a fellowship of the Télévie and $L \mathrm{~W}$ is a research director of the FNRS.

\section{Author contribution statement}

S N S performed the lab experiments and analyzed raw data. A B, E H, M $C$ and $R L$ organized trial and analyzed clinical data. M C contributed to patient recruitment and ethics. A B, E H and $\mathrm{L} W$ conceived and designed the experiments. All co-authors contributed to writing and critical reading of the manuscript.

\section{Acknowledgments}

The authors are grateful to the GIGA technological platforms in particular for the acquisition of the CellSearch as well as for support in immunohistology. The authors thank Elisei Rossella (Università di Pisa, Italy) and Karin Forsberg Nilsson (Uppsala University, Sweden) for providing cell lines, Caroline Maréchal and Stephanie Max for data managing and JeanRock Jacques and Nathalie Renotte for technical help. 


\section{References}

Alves TG, Kasamatsu TS, Yang JH, Meneghetti MCZ, Mendes A, Kunii IS, Lindsey SC, Camacho CP, Dias Da Silva MR, Maciel RMB, et al. 2016 Macrocalcitonin is a novel pitfall in the routine of serum calcitonin immunoassay. Journal of Clinical Endocrinology and Metabolism 101 653-658. (https://doi.org/10.1210/jc.2015-3137)

Hou JM, Krebs MG, Lancashire L, Sloane R, Backen A, Swain RK, Priest LJC, Greystoke A, Zhou C, Morris K, et al. 2012 Clinical significance and molecular characteristics of circulating tumor cells and circulating tumor microemboli in patients with small-cell lung cancer. Journal of Clinical Oncology 30 525-532. (https://doi. org/10.1200/JCO.2010.33.3716)

Ito Y, Miyauchi A, Kihara M, Kudo T \& Miya A 2016 Calcitonin doubling time in medullary thyroid carcinoma after the detection of distant metastases keenly predicts patients' carcinoma death. Endocrine Journal 63 663-667. (https://doi.org/10.1507/endocrj.EJ16-0140)

Krebs MG, Sloane R, Priest L, Lancashire L, Hou JM, Greystoke A, Ward TH, Ferraldeschi R, Hughes A, Clack G, et al. 2011 Evaluation and prognostic significance of circulating tumor cells in patients with non-small-cell lung cancer. Journal of Clinical Oncology 29 1556-1563. (https://doi.org/10.1200/JCO.2010.28.7045)

Kuo JH, Chabot JA \& Lee JA 2016 Breast cancer in thyroid cancer survivors: an analysis of the Surveillance, Epidemiology, and End Results-9 database. Surgery 159 23-29. (https://doi.org/10.1016/j. surg.2015.10.009)

Xing M 2013 Molecular pathogenesis and mechanisms of thyroid cancer. Nature Reviews Cancer 13 184-199. (https://doi.org/10.1038/ $\operatorname{nrc} 3431)$

Yu M, Stott S, Toner M, Maheswaran S \& Haber DA 2011 Circulating tumor cells: approaches to isolation and characterization. Journal of Cell Biology 192 373-382. (https://doi.org/10.1083/ jcb.201010021)

Yu XJ, Handy B, Michaelis CL, Waguespack SG, Hu MI, Busaidy N, Jimenez C, Cabanillas ME, Fritsche HA, Cote GJ, et al. 2016 Detection and prognostic significance of circulating tumor cells in patients with metastatic thyroid cancer. Journal of Clinical Endocrinology and Metabolism 101 4461-4467. (https://doi. org/10.1210/jc.2016-2567)

Received in final form 29 July 2018

Accepted 1 August 2018

Accepted Preprint published online 2 August 2018
(C) 2018 Society for Endocrinology Published by Bioscientifica Ltd. Printed in Great Britain 